\title{
Voigt, Miriam
}

\section{Neo-institutionalistische und mikropolitische Prozesse in}

\section{Schulentwicklungsprojekten}

Seifried, Jürgen [Hrsg.]; Faßhauer, Uwe [Hrsg.]; Seeber, Susan [Hrsg.]: Jahrbuch der berufs- und wirtschaftspädagogischen Forschung 2014. Opladen [u.a.] : Verlag Barbara Budrich 2014, S. 175-185. (Schriftenreihe der Sektion Berufs- und Wirtschaftspädagogik der Deutschen Gesellschaft für Erziehungswissenschaft (DGfE); 217)

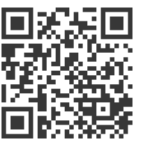

Quellenangabe/ Reference:

Voigt, Miriam: Neo-institutionalistische und mikropolitische Prozesse in Schulentwicklungsprojekten - In: Seifried, Jürgen [Hrsg.]; Faßhauer, Uwe [Hrsg.]; Seeber, Susan [Hrsg.]: Jahrbuch der berufs- und wirtschaftspädagogischen Forschung 2014. Opladen [u.a.] : Verlag Barbara Budrich 2014, S. 175-185 URN: urn:nbn:de:0111-pedocs-97348 - DOI: 10.25656/01:9734

https://nbn-resolving.org/urn:nbn:de:0111-pedocs-97348

https://doi.org/10.25656/01:9734

in Kooperation mit / in cooperation with:

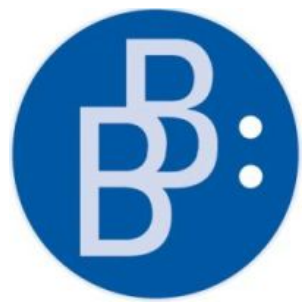

https://www.budrich.de

\section{Nutzungsbedingungen}

Dieses Dokument steht unter folgender Creative Commons-Lizenz:

http://creativecommons.org/licenses/by-nc-nd/3.0/de/deed - Sie dürfen das Werk bzw. den Inhalt unter folgenden Bedingungen vervielfältigen, verbreiten und öffentlich zugänglich machen: Sie müssen den Namen des Autors/Rechteinhabers in der von ihm festgelegten Weise nennen. Dieses Werk bzw. dieser Inhalt darf nicht für kommerzielle Zwecke verwendet werden und es darf nicht bearbeitet, abgewandelt oder in anderer Weise verändert werden.

Mit der Verwendung dieses Dokuments erkennen Sie die Nutzungsbedingungen an.

\section{Terms of use}

This document is published under following Creative Commons-License: http://creativecommons.org/licenses/by-nc-nd/3.0/de/deed.en - You may copy, distribute and transmit, adapt or exhibit the work in the public as long as you attribute the work in the manner specified by the author or licensor. You are not allowed to make commercial use of the work or its contents. You are not allowed to alter, transform, or change this work in any other way.

By using this particular document, you accept the above-stated conditions of use.

\section{Kontakt / Contact:}

\section{peDOCS}

DIPF | Leibniz-Institut für Bildungsforschung und Bildungsinformation Informationszentrum (IZ) Bildung

E-Mail: pedocs@dipf.de

Internet: www.pedocs.de

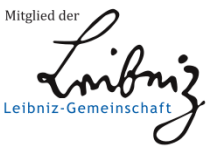




\section{Jahrbuch der berufs- und wirtschaftspädagogischen Forschung 2014}

Jürgen Seifried, Uwe Faßhauer Susan Seeber (Hrsg.)

DGE Deutsche Gesellschaft 
Schriftenreihe der Sektion

Berufs- und Wirtschaftspädagogik der Deutschen Gesellschaft für Erziehungswissenschaft (DGfE) 
Jürgen Seifried

Uwe Faßhauer

Susan Seeber (Hrsg.)

\section{Jahrbuch der berufs- und wirtschaftspädagogischen Forschung 2014}

Verlag Barbara Budrich

Opladen • Berlin • Toronto 2014 
Bibliografische Information der Deutschen Nationalbibliothek

Die Deutsche Nationalbibliothek verzeichnet diese Publikation in der Deutschen Nationalbibliografie; detaillierte bibliografische Daten sind im Internet über http://dnb.d-nb.de abrufbar.

(C) Dieses Werk ist im Verlag Barbara Budrich erschienen und steht unter folgender Creative Commons Lizenz: http://creativecommons.org/licenses/by-nc-nd/3.0/de/ Verbreitung, Speicherung und Vervielfältigung erlaubt, kommerzielle Nutzung und Veränderung nur mit Genehmigung des Verlags Barbara Budrich.

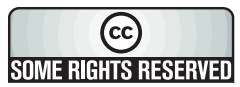

Dieses Buch steht im OpenAccess Bereich der Verlagsseite zum kostenlosen Download bereit (http://dx.doi.org/10.3224/84740164)

Eine kostenpflichtige Druckversion (Printing on Demand) kann über den Verlag bezogen werden. Die Seitenzahlen in der Druck- und Onlineversion sind identisch.

$$
\begin{array}{ll}
\text { ISBN } & 978-3-8474-0164-3 \text { (Paperback) } \\
\text { eISBN } & \mathbf{9 7 8 - 3 - 8 4 7 4 - 0 4 4 1 - 5 ~ ( e B o o k ) ~} \\
\text { DOI } & 10.3224 / 84740164
\end{array}
$$

Umschlaggestaltung: bettina lehfeldt graphic design, Kleinmachnow

Typografisches Lektorat: Judith Henning, Hamburg

Verlag Barbara Budrich, http://www.budrich-verlag.de 


\section{Inhaltsverzeichnis}

Vorwort 9

\section{Teil I: Perspektiven der historischen Berufsbildungsforschung}

Frank-Lothar Kroll

Möglichkeiten und Notwendigkeiten historiographischen Arbeitens in der Berufs- und Wirtschaftspädagogik........................................................ 1

Volker Bank, Annekathrin Lehmann

Theodor Franke. Sächsischer Pionier wirtschaftspädagogischen

Denkens in Deutschland

Marcel Schweder

Lehrerarbeit im Strafvollzug - Ein Diskurs aus historischer Sicht

\section{Teil II: Kompetenzmodellierung, -messung und -förderung}

Eveline Wittmann, Ulrike Weyland, Annette Nauerth, Ottmar Döring, Simone Rechenbach, Julia Simon, Iberé Worofka Kompetenzerfassung in der Pflege älterer Menschen - Theoretische und domänenspezifische Anforderungen der Aufgabenmodellierung 53

Simon Heinen, Martin Frenz, Christopher M. Schlick

Bildung für nachhaltige Entwicklung in der Gebäudeenergieberatung - Entwicklung eines Kompetenzmodells für die Förderung reflexiver Handlungsfähigkeit

Diana Stuckatz, Cornelia Wagner

Qualifizierungsangebote in der Pflegehilfe für Personen mit geringen Grundbildungskenntnissen - Empirische Studien zur Entwicklung von Lehr-Lern-Umgebungen und Arbeitsmaterialien. 81 


\section{Teil III: Gestaltung und Analyse von Lehr-Lern-Prozessen}

Eva Höpfer, Andrea Reichmuth, Doreen Holtsch, Franz Eberle

Wer sieht was? - Zum Umgang mit unterschiedlichen Sichtweisen auf

Unterricht am Beispiel des kaufmännischen Berufsschulunterrichts 95

Mandy Hommel

Sozial geteilte Reflexion - eine explorative Studie im

Mathematikunterricht. 109

Gerhard Minnameier, Rico Hermkes

„Kognitive Aktivierung“ und „konstruktive Unterstützung“ als Lehr-

Lern-Prozess-Größen - Eine Konzeption im rechnungswesen-

didaktischen Kontext

\section{Teil IV: Lehrerbildung und pädagogische Professionalität}

Nicole Kimmelmann, Johannes Lang

Lehramtsstudierende mit Migrationshintergrund und ihre

Schwierigkeiten an der Universität

Robert W. Jahn

Stützlehrer als neuer pädagogischer Profi in der Beruflichen Bildung?! .... 147

Sabrina Berg

Pädagogische Praxis und Reproduktion sozialer Ungleichheit - zur

Berücksichtigung sozialer Herkunft im Wirtschaftsunterricht..... 161

\section{Teil V: Perspektiven der Berufsbildungsforschung}

\section{Miriam Voigt}

Neo-institutionalistische und mikropolitische Prozesse in

Schulentwicklungsprojekten

Lara Forsblom, Lucio Negrini, Jean-Luc Gurtner \& Stephan Schumann

Lehrvertragsauflösungen und die Rolle der betrieblichen Auswahl von

Auszubildenden 
Marius R. Busemeyer

Organisierte Interessen, Parteipolitik und institutioneller Wandel im deutschen Berufsbildungssystem

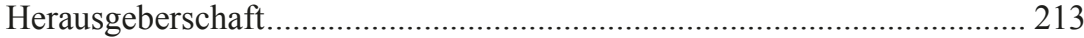

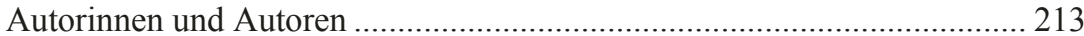




\title{
Neo-institutionalistische und mikropolitische Prozesse in Schulentwicklungsprojekten
}

\author{
Miriam Voigt
}

\section{Einleitung}

Der folgende Beitrag basiert auf der Dissertationsschrift der Autorin, welche sich mit der Analyse eines Schulentwicklungsprojekts aus neo-institutionalistischer und mikropolitischer Perspektive befasst. Ziel der Forschungsarbeit ist es, nicht-zweckrationale Prozesse in dem Projekt - mit Hilfe eines heuristischen Mehrperspektivenmodells - herauszustellen, um einen Beitrag zur Schulentwicklungstheorie leisten zu können.

Der vorliegende Artikel stellt zunächst die Herangehensweise der Dissertation im Rahmen der Schulentwicklungsforschung dar, bevor das Forschungsdesign sowie die beiden Theorien (Neo-Institutionalismus und Mikropolitik) beschrieben werden. Hierbei werden die wichtigsten theoretischen Kategorien expliziert. Diese sowie weitere entwickelte Analysekategorien, die auf den empirischen Erhebungen basieren, erfassen die nicht-zweckrationalen Prozesse des Projekts und werden schlussendlich in der Ergebnisdarstellung erläutert. ${ }^{1}$

\section{Herangehensweise}

Um die Herangehensweise der Arbeit zu legitimieren, wird als Grundlage eine umfassende Recherche im Feld der Schulentwicklungsforschung durchgeführt. $^{2}$ Bei dieser Recherche wird u. a. anhand einer Schlagwortsuche dahingehend geforscht, welche Organisationstheorien im Feld der Schulentwicklung verwendet werden. Es lässt sich zum einen feststellen, dass ein Theoriedefizit sowie ein Überhang an praxisorientierter Literatur in der Schulentwicklung existieren (vgl. hierzu Röbken 2006, S. 255ff.). Zum anderen werden meist erwerbswirtschaftlich fundierte Organisationstheorien in

1 Unter anderem diese Kategorien bilden letztlich das heuristische Mehrperspektivenmodell.

2 Bei dieser Recherche wurde in der Literaturdatenbank des Fachportals Pädagogik nach Schlagworten (u.a. Schulentwicklung, Organisationstheorie, Organisationsentwicklung, Theorien der Schulentwicklung) in Zeitschriften, Monographien und Sammelbeiträgen ab dem Jahr 1965 geforscht. 
der Schulentwicklungsforschung verwendet (z. B.: Kontingenztheorie, Bürokratiemodell, situativer bzw. kontingenztheoretischer Ansatz), welche Transferschwierigkeiten auf Schulentwicklungsprozesse implizieren, da zweckrationale Prozesse wie Ressourcen oder sachliche Faktoren im Vordergrund stehen (vgl. hierzu Warnken 2001, S. 8ff.). Auffällig ist zudem, dass die Theorie des Neo-Institutionalismus insgesamt nur zwei Mal erwähnt wird. Eine mögliche Verwendung der beiden Theorien in Kombination wird in keinem der recherchierten Werke erwähnt bzw. erläutert (vgl. Voigt 2013, S. 19ff.).

\section{Theoretische Grundlage: Neo-Institutionalismus und Mikropolitik}

Die Kombination der beiden Theorien und der damit verbundene doppelte Zugang bieten ein differenziertes Bild über Schulentwicklungsprozesse. Der Grund hierfür liegt in der spezifischen Perspektive der einzelnen Theorien. Während die Mikropolitik die Prozesse innerhalb einer Organisation auf intra-organisationaler Ebene fokussiert, legt der Neo-Institutionalismus seinen Schwerpunkt auf die Prozesse zwischen den einzelnen Organisationen und der damit verbundenen Umwelt (inter-organisationale Ebene).

\subsection{Neo-Institutionalismus}

Der Neo-Institutionalismus unterscheidet sich von den rein ökonomistischen Theorien und bezieht gesellschaftliche Aspekte sowie die institutionelle Umwelt mit in sein Erkenntnisinteresse ein. Zweckrationale Vorgänge werden nicht völlig ausgeschlossen, aber es ist festzuhalten, dass darüber hinaus sowohl kognitive Dimensionen als auch eine „kulturelle Perspektive“، ${ }^{\text {(Türk }}$ 2004, S. 924) bei der Beschreibung rationaler Ziele eine große Rolle spielen. In der Arbeit wird der umweltbezogene Neo-Institutionalismus aufgegriffen, welcher sich mit den Effekten der formalen organisationalen Strukturen und Regeln einer Organisation und den Erwartungen aus der Umwelt befasst (vgl. Walgenbach/Meyer 2008, S. 12).

In der Theorie des Neo-Institutionalismus spielen u. a. zwei Kategorien eine besondere Rolle. Dies sind zum einen die Isomorphie und zum anderen die Entkopplung von Formal- und Aktivitätsstrukturen. Die Kategorie der

3 Sowohl der Neo-Institutionalismus als auch die Mikropolitik sind Organisationstheorien und fokussieren die nicht-zweckrationale Sichtweise auf Organisationen. In der Forschungsarbeit werden die beiden Theorien im Rahmen theoriegeleiteter Kategorienschemata miteinander verknüpft. Diese Schemata bilden die Basis der empirischen Erhebungen. 
Isomorphie differenziert sich in drei verschiedene Prozesse. Zum einen ist dies die erzwungene Isomorphie, welche $u$. a. durch informellen Druck auf Organisationen entstehen kann. Grund dafür ist entweder die Interdependenz anderer Organisationen oder kulturelle gesellschaftliche Erwartungen, welche auch als Machtausübung interpretieren werden können. Darüber hinaus äußert sich die erzwungene Isomorphie meist in Vorgaben, Gesetzen oder vertraglichen Verpflichtungen (vgl. DiMaggio/Powell 1983, S. 64). Der zweite isomorphe Mechanismus ist der mimetische Prozess. Nachahmung entsteht dann, wenn Organisationen unsicher in ihren Handlungen sind und sich aufgrund dessen an den Strukturen anderer Organisationen ausrichten. Es werden jene Organisationen imitiert, die in diesem Feld als legitimer und erfolgreicher als die eigene Institution wahrgenommen werden (vgl. Galaskiewicz/Wasserman 1989, S. 476). Normativer Druck ist der dritte Mechanismus der Isomorphie und entsteht vor allem durch Professionalisierungsprozesse innerhalb einer Berufsgruppe. Als Folge entsteht „ein Pool von weitgehend austauschbaren Individuen“ (DiMaggio/Powell 1983, S. 69), die unabhängig von ihrer einzelnen Organisation gleichartige Positionen innehaben und ähnliche Orientierungen und Werte besitzen. Eine Konsequenz kann daher sein, dass diese Individuen versuchen, sich voneinander abzuheben und individuelle Wege (Weiterbildung etc.) einzuschlagen.

Die Entkopplung der Strukturen meint die Differenz zwischen Intention (Formalstruktur) und Aktivität (eigentliche Handlung) der Individuen in einer Organisation. So können sich die Mitglieder einer Organisation auf der „talk“-Ebene als innovativ, lern- und veränderungswillig zeigen, während die Aktivitätsstruktur zugleich kaum angetastet wird und „business as usual“ vorherrscht (vgl. Schaefers 2008, S. 238ff.). Diese Entkopplung der Ebenen, so Weick (1976), hat jedoch auch einige Vorteile: Beispielsweise verfügen Organisationen mit loser Kopplung über einen sensiblen Wahrnehmungsmechanismus hinsichtlich Veränderungen und können flexibel auf Modifikationen reagieren. Sie können sich folglich gut und schnell an neue Gegebenheiten anpassen und bei auftretenden Schwierigkeiten können einzelne Elemente abgekoppelt werden. In Bezug auf Schule bedeutet eine lose Kopplung der Strukturen „mehr Raum für Selbstbestimmung durch Akteure“ (Weick 1976, S. 91), da weniger zwischen den einzelnen Aktivitäten und Akteuren koordiniert werden muss und es daher seltener zu Konflikten kommt. Auch könnte eine enge Kopplung von Formal- und Aktivitätsebene in einem organisationalen Feld bedeuten, dass ggf. „Ineffizienzen und Inkonsistenzen bekannt werden würden“ (Walgenbach 2006, S. 376). Daher werden oftmals Vermeidungsstrategien eingesetzt, wie bspw. eine unklare Formulierung von Zielen, der Schutz einer Überprüfung der formalen Strukturen bzw. der Leistungsfähigkeit einer Organisation oder die Koordination auf informellem Weg (vgl. ebd.). 


\subsection{Mikropolitik}

Die Mikropolitik „fokussiert die unauffällige Feinstruktur des Handelns der verschiedenen Akteure bzw. scheinbar irrationale Vorgänge in Organisationen. Es geht ihr um eine nicht-instrumentelle Betrachtungsweise einer Innovation, in der die Ursachen für Blockaden bei Reformprozessen über Spiele und Strategien aufgeschlüsselt und legitime oder gar zweifelhafte Interessen der Akteure entdeckt werden können“ (Kuzmanovic 2003, S. 10). Akteure sind folglich in der Lage, organisationale Formal-, Ressourcen-, und Informationsstrukturen mit unterschiedlichen Taktiken und Strategien zu kontrollieren und die Handlungen anderer Akteure zu beeinflussen (vgl. Küpper/Felsch 2000, S. 151).

Auch die Organisation der Schule unterliegt einer stetigen Entwicklung. Kommt es beispielsweise zu curricularen oder organisatorischen Innovationen, divergieren die Zieloptionen hinsichtlich dieser Änderung und es entstehen $u$. U. neue Aufgaben- und Beziehungsstrukturen. Es finden sich daher auch in der Schule verschiedene Interessen der Akteure und damit verbundene Machtprozesse (vgl. Altrichter/Posch 1996). Mikropolitische Strategien können bspw. der Boykott sein, das Ausprechen von Sanktionen oder auch das Management der Außenwirkungen der Organisation. ${ }^{4}$ Des Weiteren spielen in der Mikropolitik die verschiedenen Motive der Teilnahme an einer Innovation eine wichtige Rolle. Ball (1987) identifiziert auf der Basis eigener Studien in US-amerikanischen Schulen drei Arten von Interessen bei Lehrern bezüglich mikropolitisch-machtstrategischer Prozesse bei schulischen Innovationen. Zum einen kann dies persönliches Interesse im Sinne von materiellem Streben wie beispielsweise Optimierung der Arbeitskonditionen sowie Kontrolle von Ressourcen (Zeit, Materialien und Räumlichkeiten) sein. Zum anderen kann das Interesse ideologisch orientiert sein. Darüber hinaus ist das Eigeninteresse eine Möglichkeit bezüglich der Interessenentwicklung (Charakter, Entfaltung einer individuellen Lehreridentität) (vgl. Ball 1987, S. 17).

\section{Vorstellung des Forschungsgegenstandes}

In der Forschungsarbeit wird ein Modellprojekt untersucht, an dem sich fünf berufliche Schulen beteiligen. Die einzelnen Modellprojekte befinden sich in unterschiedlichen Arbeitsphasen. Während sich einige Schulen mit den ersten konkreten unterrichtlichen Umsetzungen befassen, befinden sich andere bereits in der Optimierungsphase des Pilot-Durchgangs. Die gemeinsame kon-

4 Hier zeigt sich u. a. eine Verbindung zum Neo-Institutionalismus, der vornehmlich die Prozesse zwischen den Organisationen in den Blick nimmt. 
zeptionelle Grundlage in der Unterrichtsentwicklung besteht darin, dass alle „,individuelles Lernen in schulorganisatorisch implementierten subjektorientierten Lernumwelten“ (Kohl/Rupprecht 2008, S. 1) ermöglichen sollen. Dies bedeutet, dass die Schulen mit jeweils circa acht Lehrkräften verschiedene Projekte im Rahmen des selbstgesteuerten Lernens entwickeln. Die konkreten Konzepte sind höchst schulindividuell ausgestaltet. Das Spektrum reicht von Selbstlerntagen, die an der Pilotschule ${ }^{5}$ des Projekts durchgeführt werden, bis hin zu sogenannten individuellen Lernstunden, bei welchen die Schülerinnen und Schüler sich selbstgesteuert mit ihren Aufgaben und Skripten befassen. Insgesamt arbeiten die Schulen im Rahmen des Projektes relativ autonom an ihren Konzepten. Das zuständige Schulamt als Projektleitung nimmt dabei eine beratende Funktion ein und organisiert punktuell Fortbildungsveranstaltungen. Durch diese kommen die Schulen untereinander in Kontakt und nehmen die Chance wahr, sich gegenseitig über ihre Projekte zu informieren und sich gegenseitig Feedback zu geben. ${ }^{6}$

\section{Forschungsmethodische Anmerkungen}

Das Projekt wird mithilfe einer Einzelfallstudie innerhalb eines Zeitraums von ungefähr zwei Jahren untersucht. Lamnek erläutert in seinen Ausführungen, dass die Fallstudie multimethodisch anzulegen sei, um eine differenzierte und vielschichtige Erkenntnis sowie eine ganzheitliche Sicht auf allen Ebenen zu erreichen (vgl. 2005, S. 299). In dieser Studie werden daher Dokumentenanalysen (Analyse vorliegender Protokolle) sowie teilnehmende Beobachtungen (an Projektsitzungen) und 25 problemzentrierte Interviews mit Lehrern, Projekt- und Schulleitern sowie Mitarbeitern des Schulamts durchgeführt. Diese Erhebungen unterliegen darüber hinaus einem zirkulären Ablauf. Das heißt, dass ,die Phasen der empirischen und theoretischen Erkenntnissuche“ (Steinemann 2008, S. 94) sich einander abwechseln. Die Grundlage bilden die theoretischen Ausführungen. Diese werden durch die empirischen Erhebungen der Dokumentenanalyse, der teilnehmenden Beobachtung sowie durch die Interviews erweitert.

5 Die Pilotschule hat an dem Projekt von Beginn an mitgearbeitet und setzt ihr Konzept zum selbstgesteuerten Lernen seit dem Jahr 2006 um.

6 Des Weiteren hatte das Studienseminar für berufliche Schulen an dem Projekt teilgenommen. Die Aufgaben und Funktionen dieser Institution werden in dem vorliegenden Artikel allerdings nicht weiter fokussiert. 
Abb. 1: Zirkulärer Ablauf der empirischen Erhebung

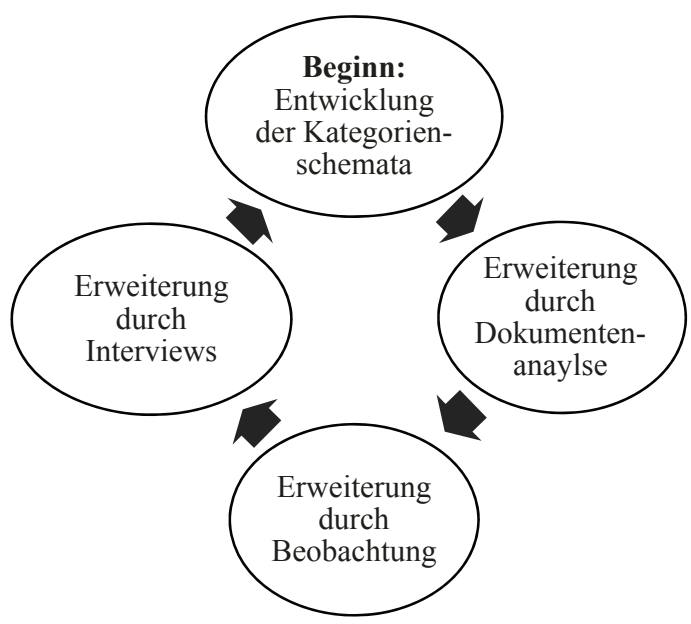

Quelle: eigene Darstellung

Der zirkuläre Ablauf des Forschungsprozesses bietet den Vorteil, dass er zu einer stetigen Reflexion des gesamten Forschungsvorgehens und seiner Zwischenschritte veranlasst. Durch die Methodentriangulation sowie den zirkulären Ablauf kann eine differenzierte und vielschichtige Erkenntnis sowie eine ganzheitliche Sicht auf allen Ebenen erreicht werden. Die Auswertung der Daten erfolgt mithilfe der qualitativen Inhaltsanalyse nach Mayring (2003).

\section{Ergebnisse}

Bereits nach Erstellung der Kategorienschemata, basierend auf den Theorien der Mikropolitik und des Neo-Institutionalismus, kann festgestellt werden, dass zum einen Verknüpfungslinien zwischen den beiden Kategorienschemata vorhanden sind sowie zum anderen die Mikropolitik auch Prozesse zwischen den Institutionen (inter-organisational) analysieren kann. Diese beiden Erkenntnisse werden nach den ersten empirischen Erhebungen (Beobachtung, Dokumentenanalyse) bestätigt. Das heuristische Mehrperspektivenmodell impliziert die intra- und interorganisationalen Prozesse und Kategorien, die eine wichtige Rolle bei der Implementierung von Schulentwicklungsprojekten spielen. Im Folgenden werden einige exemplarische Kategorien bzw. Analy- 
seergebnisse aufgeführt, welche u. a. die Hauptkategorien der beiden Theorien implizieren.

\subsection{Ergebnisse auf intra-organisationaler Ebene}

Eine mimetische Isomorphie ${ }^{7}$ innerhalb des Kreises der teilnehmenden Schulen kann nicht festgestellt werden. Die Konzepte der Pilotschule werden zwar teilweise als vorbildlich beschrieben und die Lehrer der anderen teilnehmenden Projektschulen kennen, u. a. durch die gemeinsamen Fortbildungsveranstaltungen (Kontakt der Schulen untereinander), auch die Projektstruktur, dennoch machen sie deutlich, dass die Konzepte der anderen Schulen nicht auf die eigene Schulstruktur übertragbar sind. Außerhalb dieses Projektkreises werden einige erfolgreiche Schulen genannt, aus denen Komponenten verschiedener innovativer Konzepte im Rahmen des selbstgesteuerten Lernens übernommen werden. Allerdings kann hier nicht von einer vollständigen Imitation gesprochen werden. Des Weiteren orientieren sich einige Lehrer an theoretischen Konzepten und beschreiben oftmals, dass eine sogenannte „Eigenkreation aus erworbenem Wissen“ (vgl. Voigt 2013, S. 244) in dem Projekt Anwendung findet.

Eine erzwungene Isomorphie lässt sich in den Motiven der Teilnahme erkennen. Die Schulen bzw. Lehrer nehmen u. a. auch aufgrund der gesellschaftlichen Erwartungen an dem Projekt teil. Staatliche Vorgaben, Erlasse, Handreichungen und Empfehlungen der Schulministerien sowie öffentliche staatliche Reformprojekte im Rahmen der Schule als lernende Organisation, setzen die Schulen einem hohen Legitimations- und Veränderungsdruck aus (vgl. DiMaggio/Powell 1983, S. 62f.). Weitere beschriebene Motive der Teilnahme sind die innere Überzeugung (ideologisches Interesse) oder die erhoffte Arbeitserleichterung (persönliches Interesse) (s. Ball 1987, S. 17).

Auch ein normatives isomorphes Motiv kann festgestellt werden. Da einige Lehrer aus karrierebezogenen Interessen besonders motiviert sind und gleichzeitig wissen, dass die Schulleitung dieses Projekt forciert, nehmen sie an jenem teil, um die Möglichkeit einer Beförderung gegenüber anderen Kollegen zu erhöhen (vgl. Voigt 2013, S. 214f.).

Darüber hinaus werden mikropolitische Prozesse auf intra-organisationaler Ebene deutlich. Das Projekt impliziert bspw. unter anderem eine neue Arbeits- und Beziehungskultur. Die Lehrer beschreiben, dass sie vermehrt in Teams zusammenarbeiten und so das „Einzelkämpfertum“ in positiver Hinsicht minimiert wird. In diesen Teams kommen verschiedene Lehrer zusammen und bringen unterschiedliche Projektideen ein. Auch die Beziehungsstruktur $\mathrm{zu}$ den nicht-teilnehmenden Kollegen spielt für die Lehrer im Projekt eine große Rolle. Die Akzeptanz des Projekts im Kollegi-

7 Die kursiv gedruckten Begriffe zeigen exemplarisch die entwickelten Analysekategorien. 
um ist als niedrig einzustufen: Viele Lehrer wollen ihre eigene Arbeitsbelastung schützen und nehmen deshalb nicht an dem Projekt teil, andere wiederum wollen nicht, dass ihre Arbeitsweisen im Unterricht „öffentlich“ in ein Team eingebracht werden. Ein Teil der Kollegen hinterfragt deutlich die Sinnhaftigkeit des Projekts, da dies nicht ihren Vorstellungen bzw. Normen und Werten von Schule und Unterricht entspricht. Diese Blockaden zeigen sich in Strategien des Desinteresses und in ironischen bzw. sarkastischen Bemerkungen. Der mikropolitische Begriff der Macht wird in der Analyse an den Besitz von zusätzlicher Ausstattung sowie Unterstützung der Schulleitung gekoppelt. Die beteiligten Lehrer fühlen sich in dieser Hinsicht bevorteilt gegenüber nicht-teilnehmenden Kollegen. ${ }^{8}$ Letztlich ist es daher eine wichtige Aufgabe der Lehrer und auch der Schulleiter durch transparente Handlungen die Beziehungsstruktur zu ihren Kollegen aufzuarbeiten.

\subsection{Ergebnisse auf inter-organisationaler Ebene}

Eine Entkopplung der Formal- und Aktivitätsstruktur kann hinsichtlich der intra-organisationalen Projektarbeit in den Schulen nicht bekräftigt werden. Es finden vielseitige Veränderungen auf der ,action-Ebene“ statt, die durch die beteiligten Lehrer initiiert werden. Dies wird in der Beschreibung der Lehrer hinsichtlich der neuen Lehr-/Lernarrangements in den einzelnen Schulen deutlich. Ebenso sind organisationale Strukturen wie bspw. regelmäßige Teamsitzungen, Personaländerungen, Neuregelungen hinsichtlich der Räume und Stundenpläne, eine generelle Ausstattung mit Ressourcen, reformiert.

Auf der inter-organisationalen Ebene zwischen dem Schulamt und den Schulen kann allerdings eine solche Entkopplung festgehalten werden (Rolle des Schulamts). ${ }^{9}$ Die Schulen (= Aktivitätsebene) arbeiten an ihren Projekten und nehmen Veränderungen vor. Das Schulamt (= Formalebene/Projektleitung) hingegen arbeitet lediglich punktuell mit den Schulen zusammen. Die Intention der Projektleitung impliziert allerdings auch eine kontinuierliche Unterstützung an den Schulen, kann dies allerdings durch die geringen Ressourcen nicht wie geplant einhalten. Zudem weisen die verschiedenen Ausprägungen bzw. Strukturen der einzelnen Projekte in den Schulen auf eine Entkopplung der Formal- und Aktivitätsstruktur auf inter-organisationaler Ebene hin (s. DiMaggio/Powell 1983, S. 64). Nach Weick kann eine lose

8 Darüber hinaus konnten in diesem Zusammenhang weitere mikropolitische Handlungen wie Grenzziehungen zwischen Einflussbereichen, Tratsch und Gerücht, Management der Außenwirkungen, positive Sanktionen wie Wertschätzung und materielle Ressourcen, Boykott, Eskalation sowie Aufbau von Bindungen und Vertrauen (s. Altrichter/Posch 1996, S. 106ff.) festgestellt werden.

9 Diese Entkopplung zwischen den Organisationen geht über die beschriebene Entkopplung der Formal- und Aktivitätsebene auf Basis der Theorie des Neo-Institutionalimsus hinaus und kann als ein wichtiges Ergebnis der Arbeit definiert werden. 
Kopplung der Strukturen allerdings auch „mehr Raum für Selbstbestimmung" (1976, S. 91) bedeuten. Durch die Entkopplung der beiden Ebenen haben die einzelnen Schulen einen höheren Gestaltungsspielraum, welcher möglicherweise auch das Konfliktpotential minimiert. Dieser Gestaltungsspielraum innerhalb der einzelnen Projekte wird sowohl von den Lehrern als auch von den Schulleitungen als positiv wahrgenommen.

\section{Fazit}

Die Erkenntnisse aus den empirischen Erhebungen zeigen sowohl auf der intra-organisationalen als auch auf der inter-organisationalen Ebene des Projekts vielfältige Ergebnisse. Die beiden Theorien nehmen eine nicht zweckrationale Perspektive ein und bieten somit einen ergänzenden bzw. erweiternden Blick auf die Schulentwicklungsforschung (siehe Abschnitt 2). Die Mikropolitik bezieht sich schwerpunktmäßig auf die Akteure und deren Beziehungs- und Handlungsstrukturen zwischen den einzelnen Institutionen innerhalb des Projekts. Die externen Beziehungen sowie die gesellschaftliche und politische Einbettung der Schulen sowie des Projekts werden vornehmlich von der Theorie des Neo-Institutionalismus betrachtet. Somit werden die nicht zweckrationalen und komplexen Prozesse der Projektimplementierungen offengelegt und relevante Kriterien für Projektimplementierungen (Motive der Teilnahme, mikropolitische Prozesse, Rolle des Schulamts, Kontakte der Schulen untereinander) erläutert.

Die hier exemplarisch erläuterten Analysekategorien finden sich letztlich in dem erstellten heuristischen Mehrperspektivenmodell wieder, welches sowohl auf Praxis- als auch auf Theorieebene Leistungen erbringt. Hinsichtlich der Praxisebene können Schlussfolgerungen für die Schulen und das Schulamt als Projektleitung gezogen werden. Diese lassen wiederum Handlungsempfehlungen für die teilnehmenden Institutionen $\mathrm{zu}$. Beispielsweise zeigen die Ergebnisse die Relevanz der Teilnahmemotive der einzelnen Lehrer, auf die besonders bei Projektimplementierungen zu achten ist. Auf der Ebene der einzelnen Schulen können die Ergebnisse der empirischen Erhebungen für Schul- und Unterrichtsentwicklungen genutzt werden, um innerschulischen Diskussionsprozessen Impulse zu geben und die diagnostischen Fähigkeiten der Lehrkräfte zu stärken (vgl. Hartung-Beck 2009, S. 9). Hier sind beispielhaft die Relevanz der Teamarbeit und die mikropolitischen Prozesse zu erwähnen.

Auf theoretischer Ebene kann das Modell einen Beitrag zur Schulentwicklungstheorie leisten, indem es die beiden Theorien auf neue Art und Weise miteinander verknüpft und auf das Projekt anwendet sowie die nichtzweckrationalen Prozesse und deren Bedeutung analytisch hervorhebt. Des 
Weiteren kann das Modell als Basis weiterer Modellversuchsforschungen bspw. für das Governance-Konzept oder die Thematik der Professionalisierungsprozesse der Lehrer dienen. Es kann bspw. die Frage gestellt werden, inwieweit die Professionalisierung der Lehrer durch Reflektion des eigenen Wissenstandes oder der eigenen Rolle in den Projektstrukturen gestaltet werden kann (vgl. Sloane 2005, o. S.).

\section{Literatur}

Altrichter, H./Posch, P. (Hrsg.) (1996): Mikropolitik der Schulentwicklung. Innsbruck: Studien Verlag.

Ball, S. (1987): The micropolitics of the school: towards a theory of school organization. London: Methuen.

DiMaggio, P. J./Powell, W. W. (1983): Das ,stahlharte Gehäuse' neu betrachtet: Institutionelle Isomorphie und kollektive Rationalität in organisationalen Feldern. In: Koch, S./Schemmann, M. (Hrsg.) (2009): Neo-Institutionalismus und Erziehungswissenschaft. Wiesbaden, S. 57-85. Original: The Iron Cage Revisited: Institutional Isomorphism and Collective Rationality in Organizational Fields. In: American Sociology, H. 48, S. 147-160.

Galaskiewicz, J./Wasserman, S. (1989): Mimetic Processes Within an Interorganizational Field: An Empirical Test. In: Administrative Science Quarterly, H. 3, S. 454-479.

Hartung-Beck, V. (2009): Schulische Organisationsentwicklung und Professionalisierung. Wiesbaden: Springer.

Kohl, M./Rupprecht, E. (2008): Projektantrag und Projektkonzeption für das Entwicklungsprojekt. Nicht veröffentlichtes Dokument.

Küpper, W./Felsch, A. (2000): Organisation, Macht und Ökonomie. Wiesbaden: Verlag für Sozialwissenschaften.

Kuzmanovic, R. (2003): Lernfelder, Implementationstheorien und mikropolitische Mechanismen. In: bwpat, H. 4. http://www.bwpat.de/ausgabe4/kuzmanovic bwpat4.pdf (07-06-2011).

Lamnek, S. (2005): Qualitative Sozialforschung. Weinheim und Basel.

Mayring, P. (2003): Qualitative Inhaltsanalyse. Weinheim und Basel.

Röbken, H. (2006): Managementkonzepte in der Schulentwicklung- Eine Modeerscheinung? In: Zeitschrift für Erziehungswissenschaft. H. 2, S. 255-271.

Schaefers, C. (2008): Steigerungssemantiken im Organisationsentwicklungsdiskurs. Neo-institutionalistische Perspektive auf Konsequenzen für Schule und Lehrerprofessionalität. In: Helsper, W. et al. (Hrsg.): Pädagogische Professionalität in Organisationen. Wiesbaden: Springer, S. 325-343.

Sloane, P. F. E. (2005): Innovationen in schulischen Kontexten: Ansatzpunkte für berufsbegleitende Lernprozesse bei Lehrkräften. www.bwpat.de/spezial2/sloane. html. (25-05-2012). 
Steinemann, S. (2008): Strukturen und Prozesse von Lehrerteamarbeit im Kontext der Lernfeldumsetzung: Entwicklung eines kategorialen Analysemodells auf der Grundlage einer Fallstudie. Paderborn: Eusl.

Türk, K. (2004): Neoinstitutionalistische Ansätze. In: Schreyögg, G./Werder, A./Türk, K. (Hrsg.): Handwörterbuch Unternehmensführung und Organisation. Stuttgart: Schäffer-Poeschel, S. 923-931.

Voigt, M. (2013): Schulentwicklungsprojekte aus neo-institutionalistischer und mikropolitischer Perspektive. Eine theorieorientierte Modellentwicklung am Beispiel einer Einzelfallstudie. Kassel: Kassel University Press.

Walgenbach, P. (2006): Die Strukturationstheorie. In: Kieser, A./Ebers, M. (Hrsg.): Organisationstheorien. Stuttgart: Kohlhammer, S. 403-426.

Walgenbach, P./Meyer, R. (2008): Neoinstitutionalistische Organisationstheorie. Stuttgart: Kohlhammer.

Warnken, G. (2001): Organisationsentwicklung - ein Auslaufmodell? In: SchulManagement. H. 5, S. 7-12.

Weick, K. E. (1976): Bildungsorganisationen als lose gekoppelte Systeme. In: Koch, S./Schemmann, M. (Hrsg.) (2009): Neo-Institutionalismus und Erziehungswissenschaft, S. 85-110. Original: Educational Organizations as Loosely Coupled Systems. In: Administrative Science Quarterly, H. 21, S. 1-19. 